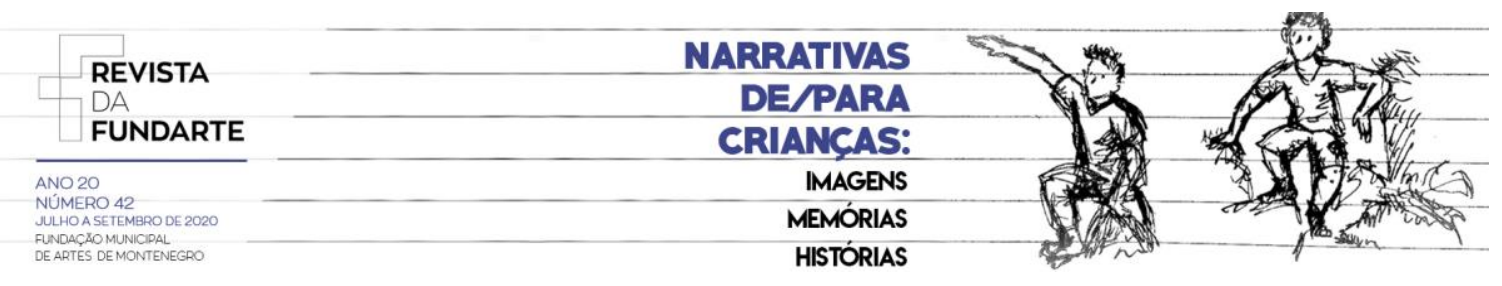

\title{
C'EST CHAUD...LA CRÉATION DANS DES ZONES DANGEREUSES
}

Maria da Graça Muniz Lima

DOI: 10.19179/2319-0868.800 Montenegro, p.01-20, ano 20, no 42, julho/setembro de 2020.

Disponível em: http://.seer.fundarte.rs.gov.br/index.php/RevistadaFundarte/index> 30 de setembro de 2020 


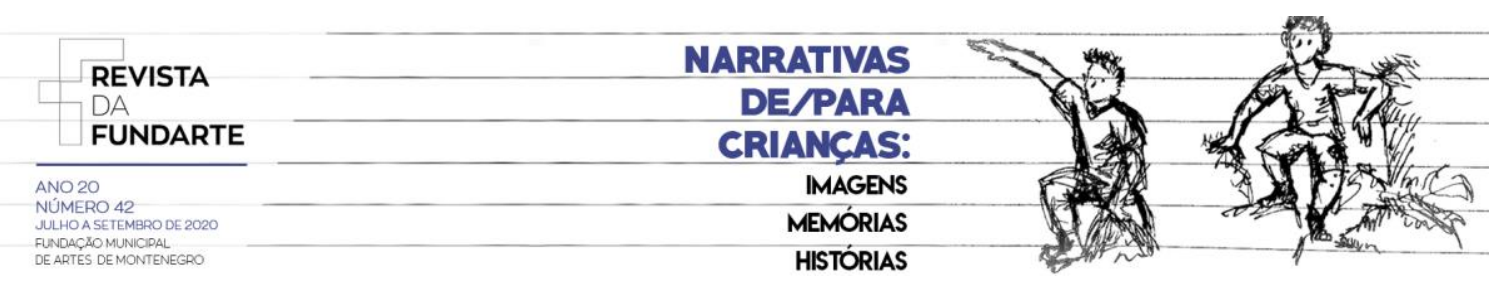

\section{C’EST CHAUD...LA CRÉATION DANS DES ZONES DANGEREUSES}

Maria da Graça Muniz Lima'

Résumé: Rio de Janeiro, la "cité merveilleuse", connue dans le monde entier pour sa beauté naturelle, se caractérise également par des contrastes sociaux extrêmes. La vie dans des bidonvilles, marginalisés par la violence croissante, franchit les frontières géographiques et gagne le monde par la voix des médias. Dans ce paysage, les enfants sont exposés à des situations de risque extrême qui s'entremêlent avec d'autres expériences, construisent des concepts ainsi que leur vision du monde. Dans les écoles publiques desservant les enfants de ces bidonvilles, la violence déborde le quotidien qui s'imprime dans leurs imaginations. Chez eux, ces éléments se combinent avec des ingrédients de l'imaginaire construisant des récits culturels riches d'expressivité. Penser l'enfant dans ce contexte particulier est un défi qui a pris de l'importance avec des recherches récentes sur l'enfance dans la scène brésilienne. Cet article présente le dessin comme un outil de construction contribuant au processus de structuration de la pensée et du regard et stimulant la créativité et la liberté de pensée. Notre réflexion prend appui sur un atelier d'image narrative développé au sein du projet "Seconde période culturelle » (Segundo Turno Cultural), qui desservait le programme "Écoles de demain » (Escolas do Amanhã). Les cas décrits ont eu lieu dans deux écoles municipales de la ville de Rio de Janeiro. La première est l'École Julia Lopes, située dans le quartier de Santa Teresa, dont la plupart des élèves proviennent d'un bidonville du Morro dos Prazeres. La seconde est l'École Anne Frank, située dans l'enceinte du Palais Guanabara, siège du gouvernement de l'État de Rio de Janeiro, dans le quartier aisé de Laranjeiras. Ces élèves-ci sont issus de familles travaillant dans le commerce et dans les condomínios (spèce des villas) de la région.

Mots-clés: Création; Enfant; Zones dangereuses.

\section{É FOGO... PROCESSO CRIATIVO EM ÁREAS DE RISCO}

Resumo: O Rio de Janeiro, Cidade Maravilhosa, conhecida no mundo inteiro por suas belezas naturais, é também caracterizada por contrastes sociais extremos, onde a vida em comunidades de baixa renda, marginalizadas pela crescente violência, ultrapassa os limites geográficos e ganha o mundo pela voz da mídia. Nesse cenário, crianças vivenciam situações de extremo risco que se entrelaçam com demais experiências e constroem conceitos e sua visão de mundo, impactadas pelo meio em que vivem. Nas escolas públicas que atendem crianças dessas comunidades, a violência ultrapassa o cotidiano e cria registros cruzados com o imaginário em narrativas culturais

1 Professora da Escola de Belas Artes da UFRJ. Designer, ilustradora e autora de livros infanto-juvenis, já tendo publicado mais de 100 obras. Ganhou vários prêmios com seu trabalho, entre eles os da FNLIJ Prêmio Luis Jardim, Prêmio Malba Tahan, Prêmio $O$ Melhor para o Jovem e muitos Altamente Recomendável. Foi indicada entre os finalistas para o prêmio Jabuti muitas vezes e recebeu em 1982, 1984 e 2003 este prêmio na categoria de ilustração. Fora do Brasil recebeu quatro vezes a Menção White Ravens da Biblioteca de Munique na Alemanha. Alguns de seus trabalhos já viajaram por outros países e foram publicados em catálogos Internacionais como o Catálogo de llustradores da Feira de Barcelona, na Espanha; 0 da Feira de Frankfurt, na Alemanha, o Catálogo da Feira da Brastslávia; e o Catálogo Brazil a Bright Blend of Colours, feito pela FNLIJ para divulgar o trabalho dos ilustradores brasileiros.

LIMA, Maria da Graça Muniz. C'est chaud...la création dans des zones dangereuses. Revista da FUNDARTE. Montenegro, p.01-20, ano 20, no 42, julho/setembro de 2020.

Disponível em: http://.seer.fundarte.rs.gov.br/index.php/RevistadaFundarte/index> 30 de setembro de 2020 


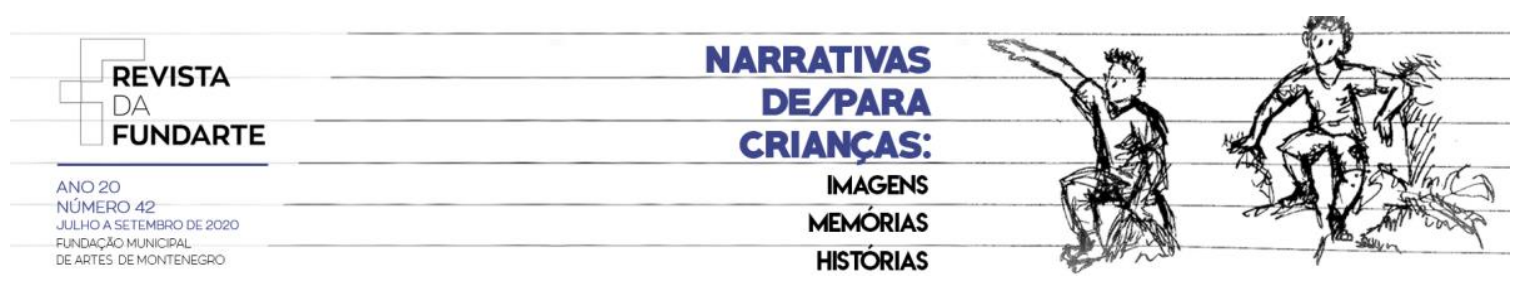

ricas de expressividade. Pensar a criança, nesse contexto específico, é um desafio que ganhou destaque nos últimos anos com pesquisadores da infância dentro do cenário brasileiro. Este artigo apresenta o desenho como ferramenta de construção, que contribui no processo de estruturação do pensamento e do olhar estimulando a criatividade e a liberdade de pensar. Nossa reflexão parte do resultado da uma oficina de imagem narrativa, desenvolvida no projeto "Segundo Turno Cultural", que atendia o "Programa Escolas do Amanhã". Os casos relatados se deram na escola municipal Julia Lopes, localizada em Santa Teresa, com alunos em sua maioria oriundos da comunidade do Morro dos Prazeres, e na escola municipal Anne Frank, situada dentro do terreno do Palácio Guanabara, sede do governo do estado, no bairro das Laranjeiras, onde os alunos pertencem a famílias de prestadores de serviços no comércio e condomínios da região.

Palavras-chave: Processos de criação; Crianças; Zona de risco.

\section{IT'S HARD... CREATIVE PROCESS IN RISK ZONES}

Abstract: Rio de Janeiro, the Wonderful City, known throughout the world for its natural beauties, is also characterized by extreme social contrasts, where life in low-income communities, which are marginalized by growing violence, surpasses geographical boundaries and gains the world through the voice of the media. In this scenario, children experience situations of extreme risk that are intertwined with other experiences and develop their concepts and views of the world, impacted by the environment in which they live. In public schools that serve children in these communities, violence goes beyond everyday life and creates records that are crossed with the imaginary in highly expressive cultural narratives. Looking at children in this specific context is a challenge that has, in recent years, been in the foreground of childhood researchers in the Brazilian scene. This article presents drawing as a tool for narrative construction, which contributes to the process of structuring the mindset and the views by stimulating creativity and free thinking. Our reflection derives from the outcomes of a workshop on narrative image, developed in a project called Segundo Turno Cultural (Second Cultural Round), in the realm of a program called Escolas do Amanhã (Schools of Tomorrow). The cases herein took place in the Julia Lopes Municipal School, located in the district of Santa Teresa, with students who come mostly from the Morro dos Prazeres community, and in the Anne Frank Municipal School, located in grounds of the State Government's Palace of Guanabara, in the district of Laranjeiras, whose students come from families of local retailers and service providers.

Keyswords: Creative process; Crildren; Risk zones.

\section{Rio quarante degrés ${ }^{2}$}

Cité merveilleuse 3

2 N.D.T. Le vers de la chanson éponyme fait référence au titre du film de Nelson Pereira dos Santos sorti en 1956: Rio, $40^{\circ}$.

LIMA, Maria da Graça Muniz. C'est chaud...la création dans des zones dangereuses. Revista da FUNDARTE. Montenegro, p.01-20, ano 20, no 42, julho/setembro de 2020.

Disponível em: http://.seer.fundarte.rs.gov.br/index.php/RevistadaFundarte/index> 30 de setembro de 2020 


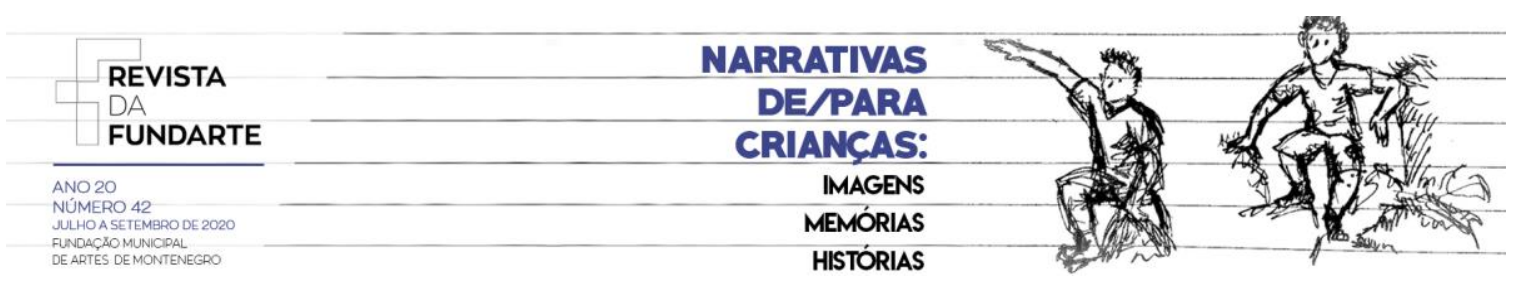

Purgatoire de la beauté et du chaos

Capitale du sang chaud du Brésil

Capitale du sang chaud

Du meilleur et du pire du Brésil

Ville au sang chaud

Merveille mouvante

Rio est une ville de villes mélangées

Rio est une ville de villes camouflées

Avec des gouvernements mélangés, camouflés, parallèles

Rio 40 degrés - Fernanda Abreu/Fausto Fawcett/ Laufer

\section{Le purgatoire de la beauté et du chaos - la construction de la symbolisation}

La discussion sur l'importance de l'éducation artistique présuppose un débat philosophique qui implique la conceptualisation de ce qui est l'homme. Nous trouvons dans la pensée d'Ernst Cassirer des éléments pour examiner cette question. Cassirer comprend l'homme comme un animal symbolique, qui organise son expérience par l'intermédiaire de symboles. Ceux-ci sont des formes de sentir et d'élaborer. Le philosophe propose, dans son Essai sur l'homme (2005 [1944], p. 49), la conception de l'homme en tant qu'animal symbolicum, corrigeant et élargissant la définition classique de l'homme en tant animal rationnel. Cette étude résulte d'une analyse sur les façons dont le monde de l'expérience humaine se façonne de différentes manières propres à la pensée humaine, c'est-à-dire, par la symbolisation.

Cette redéfinition de la nature humaine sous-entend une théorie de la connaissance qui fonde et explique les formes de connaissance du monde. Celles-ci imprègnent toutes les dimensions de l'action humaine, permettant un rapprochement entre les formes de connaissance de la science et de l'art.

La science et l'art, en tant que formes symboliques, peuvent être compris comme des formes de connaissance de la réalité. La présence de ceux-ci dans l'expérience

3 N.D.T. : Le surnom de Rio de Janeiro est « Cidade Maravilhosa », en français : « cité merveilleuse ». 


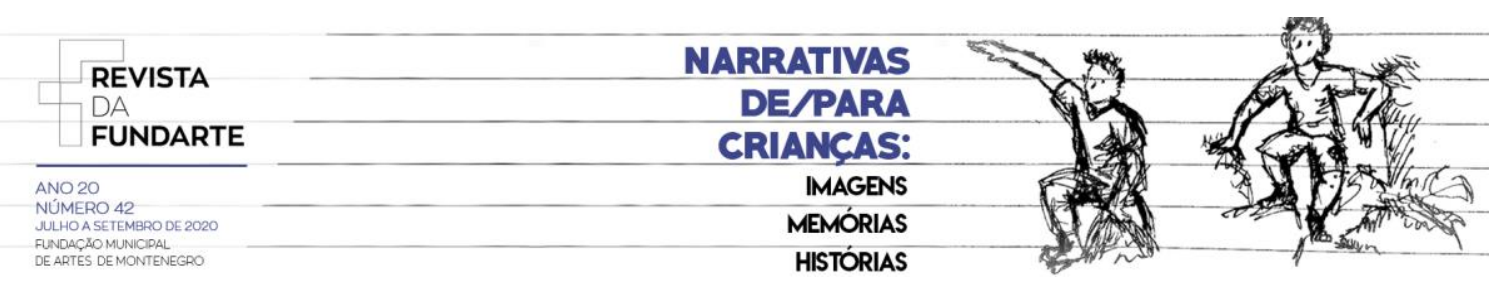

humaine montre que nous sommes en mesure de modifier notre vision de la réalité. Néanmoins, la conscience de la profondeur des choses requiert un effort de nos énergies actives et de nos énergies constructives. Présentant différents aspects de la réalité, ces deux types d'énergie ne s'orientent pas dans la même direction ni dans le même but. Dans la structuration de la connaissance, il existe une profondeur conceptuelle et une profondeur visuelle. Celle-là est découverte par la science et nous aide à comprendre la raison des choses; celle-ci est dévoilée par l'art et nous aide à comprendre ses formes.

Dans l'Essai sur l'homme, Cassirer analyse les principales formes symboliques de l'activité humaine : le langage, le mythe, l'art, la religion, l'histoire et la science. Dans le chapitre consacré à l'art, il postule que le langage et l'art oscillent constamment entre deux pôles opposés : l'un objectif et l'autre subjectif. Nulle théorie du langage ou de l'art ne pourrait oublier ou supprimer ces deux polarités, bien que l'accent pourrait être mis sur l'un ou l'autre. Le symbole est la clé pour comprendre la nature humaine : «[...] le processus de connaissance est à l'origine de la capacité inhérente à l'homme, qui le distingue des animaux, d'organiser son expérience à travers des symboles. » 2005, p. 49).

Le langage et l'art sont ainsi regroupés au même titre dans la catégorie de l'imitation, leur fonction principale étant mimétique. Le langage trouve son origine dans l'imitation des sons et l'art, des choses extérieures. L'imitation est un instinct fondamental, une disposition irréductible de la nature humaine. "L'imitation, affirme Aristote, est une tendance naturelle pour l'homme dès l'enfance; l'un des avantages de l'homme sur les animaux inférieurs est d'être la créature la plus imitative dans le monde et d'apprendre d'abord par l'imitation. »(1909, p.226). Même dans les situations dans lesquelles l'image provoque un certain malaise, il y a un plaisir à l'observer pour sa capacité détaillée de reproduire la réalité en tant que représentation artistique. Aristote décrit ce plaisir comme une expérience d'apprentissage par les sens - ce qui implique aussi l'esthétique. Montenegro, p.01-20, ano 20, no 42, julho/setembro de 2020.

Disponível em: http://.seer.fundarte.rs.gov.br/index.php/RevistadaFundarte/index> 30 de setembro de 2020 


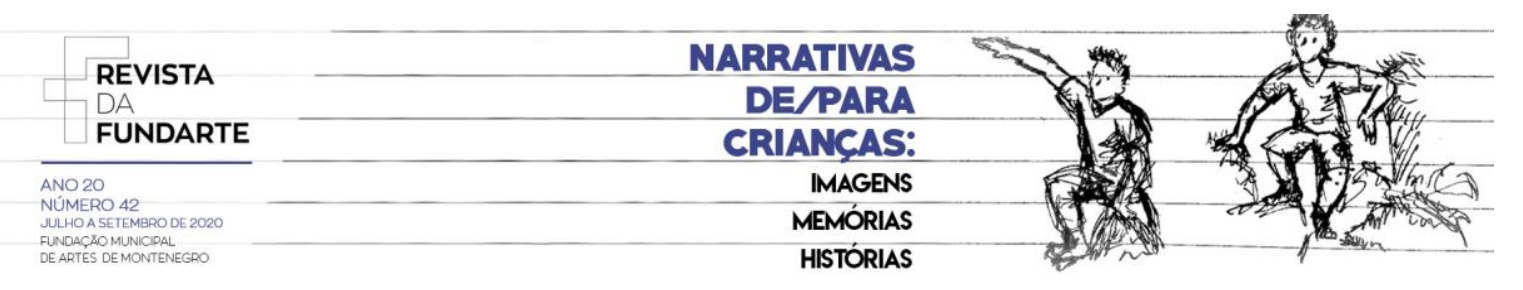

En 2009, le secrétariat de l'éducation de la municipalité de Rio de Janeiro a établi un accord avec l'UNESCO pour la mise en œuvre du "Programme Écoles de demain » (Programa Escolas do Amanhã). L'initiative touchait 150 écoles situées dans des zones à risque de Rio de Janeiro. Le programme visait à améliorer la réussite scolaire et à réduire les taux de décrochage. Dans le but d'attirer les familles et la communauté et de prévenir la violence, les écoles fonctionnaient à temps plein, proposant plusieurs activités culturelles parascolaires dans la période supplémentaire 4 .

En 2010, le secrétariat municipal de la culture, en partenariat avec le secrétariat de l'éducation, lance ainsi le projet "Seconde période culturelle» (Segundo Turno Cultural). II s'agissait de proposer des ateliers culturels parascolaires de musique, danse, théâtre, lecture et récits audiovisuels aux élèves des "Écoles de demain » ainsi qu'à 15 collèges situés à proximité de bibliothèques municipales.

Parmi les cours proposés figurait l'atelier «Raconter et illustrer» (Contando e Ilustrando) qui visait à élaborer des registres graphiques créés collectivement par chaque groupe.

Les espoirs et les craintes, les illusions et les déceptions de la vie quotidienne, étaient apportés dans leurs fantasmes et rêves. Cassirer (2005) postule que l'homme se surprend plus avec ses opinions et ses fantasmes qu'avec les faits réels, ce qui élargit la définition classique de l'homme. En effet, dans nos ateliers, il a été possible de constater à plusieurs reprises que la violence de la vie quotidienne était naturalisée par les élèves tandis que l'univers fictionnel de la télévision attendait des degrés de discussion, modifiant leurs opinions et leurs visions du monde.

L'influence des stéréotypes raciaux et sociaux dans la construction du regard figurait fréquemment en classe. Au cours d'un exercice de dessin, dans lequel chaque enfant devrait faire un autoportrait, un groupe de filles noires et métisses se sont dessinées comme des princesses blondes aux yeux bleus et la peau rosée. Interrogées

4 N.D.T. Au Brésil, les élèves fréquentent l'école pendant une période choisie : matinée, après-midi ou soirée. 


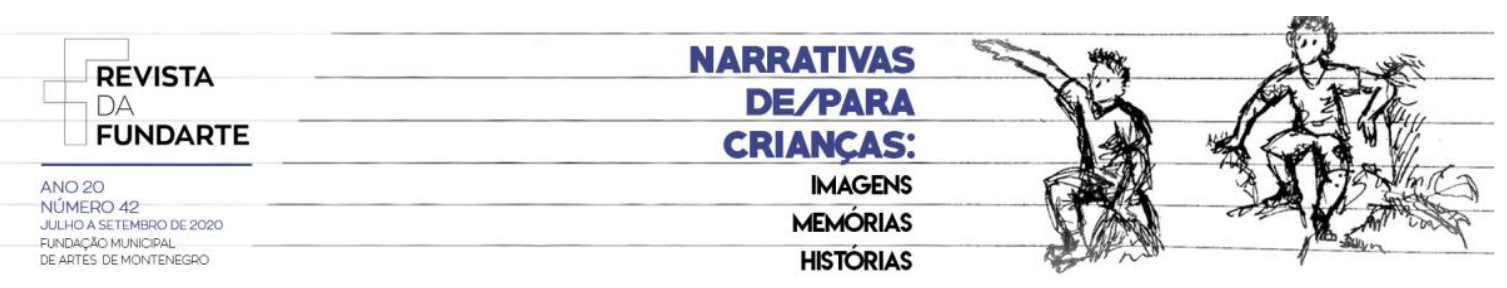

sur leurs dessins, les filles ont expliqué qu'elles cherchaient à se représenter "belles », car elles se trouvaient laides telles qu'elles étaient.

Ce gouffre socioculturel qui écarte les noirs du reste de la population brésilienne découle de la période de l'esclavage qui a forgé une vision dépréciative de l'esthétique noire, ce que la communauté noire tente de défaire aujourd'hui. Dans la préface de «Tornar-se Negro » (Devenir noir), Jurandir Freire Costa affirme que l'expérience d'être noir est celle d'être "constamment violé, de façon continue et cruelle, sans pause ou repos, par une double injonction: celle d'incarner les idéaux physiques et psychologiques du sujet blanc et celle d'annuler la présence du corps noir ». (SOUZA, 1983, p 2).

Considérant l'enfant comme un être qui construit et crée de la culture, l'acte de jouer présuppose un apprentissage social, car on y apprend le jeu des relations adultes. De cette manière, la réalité se reflète dans la version créée par les élèves du jeu/comptine brésilien «Quem roubou o pão » (Qui a volé le pain ?)5. lago a volé du pain de chez Jean, a piqué dans le frigo et a pris un coup à la main. Le refrain était chanté en funk carioca, ce qui rend la violence risible... ${ }^{6}$

On peut dire que les jeux sont la ré-élaboration créative de ce que les enfants connaissent. Cette version de la comptine confirme la pensée de certains auteurs, y compris Vygotski (2009), qui défendent que l'imagination est une combinaison de ce que les enfants connaissent avec de nouvelles façons de penser à la réalité. Tel est certainement le principe agissant dans la récréation de la comptine conjuguée avec la violence de l'entourage des enfants.

5

N.D.T. II s'agit d'une comptine où un chœur accuse l'un des enfants (« Machin a volé du pain de chez Jean»). L'enfant répond: "Qui ? Moi ? ». Le chœur répond «Toi même!». "Pas moi!» - chante l'enfant en se défendant. "Alors qui ?» — interroge le chœur. "Untel!» — chante l'accusé en 6 choisissant un autre enfant du groupe.

N.D.T. Le « funk carioca » est un genre musical originaire des favelas de Rio de Janeiro.

LIMA, Maria da Graça Muniz. C'est chaud...la création dans des zones dangereuses. Revista da FUNDARTE. Montenegro, p.01-20, ano 20, no 42, julho/setembro de 2020.

Disponível em: http://.seer.fundarte.rs.gov.br/index.php/RevistadaFundarte/index> 30 de setembro de 2020 


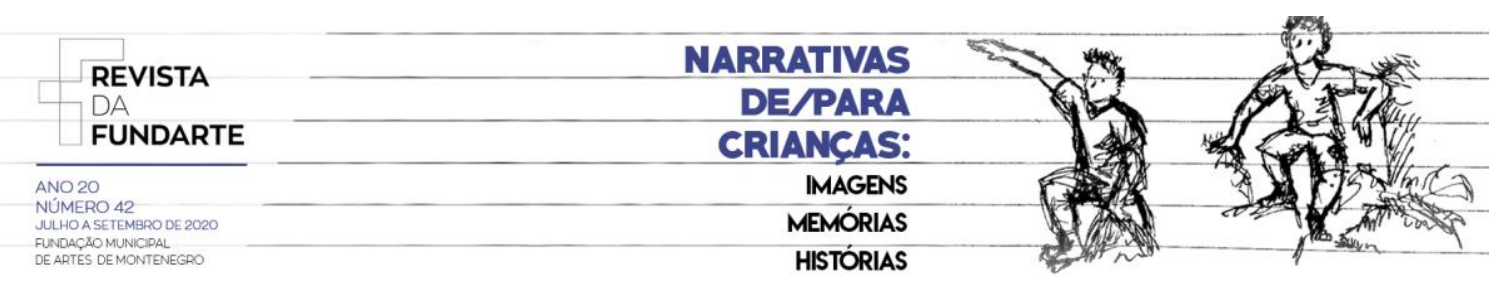

En ce qui concerne les dessins en tant que forme de reproduction de la réalité, la construction de la connaissance ne se donne pas seulement par la reconstitution interne de l'image en vue d'une reproduction fidèle, mais aussi par l'incorporation des caractéristiques des objets. Elle implique également un mouvement ambigu, qui se transforme dialectiquement lorsque les adultes et les enfants se réapproprient le jeu luimême, créant et produisant de nouvelles connaissances.

Betty Edwards, plasticienne, docteur en arts et professeur de design à la California State University, a développé une méthode qui encourage l'utilisation de I'hémisphère droit du cerveau. Souvent endormi chez la plupart des gens, son utilisation provoque une libération de la pulsion artistique, ouvrant un vaste champ de possibilités.

Lorsqu'on dessine, on utilise l'hémisphère droit du cerveau qui traite les informations d'une manière différente. II est rarement utilisé et souvent obscur. Le dessin conduit à un raisonnement créatif et intuitif. Favorisant le développement des attributs de l'hémisphère gauche, la manière traditionnelle par laquelle l'école travaille ne stimule pas suffisamment la vision spatiale des élèves. Cette préférence implique des difficultés dans l'apprentissage de certains contenus qui requièrent les compétences de l'hémisphère droit. Le langage verbal est un système symbolique fondamental, mais il n'est pas le seul. Puisque l'école valorise seulement le système de langage oral ou écrit, elle n'ouvre pas la possibilité aux expériences qui peuvent élargir la compétence symbolique. L'enfant quittera le dessin s'il n'y trouve pas de plaisir et s'il n'est pas autorisé à explorer sa fonction expressive et son potentiel créatif. II est nécessaire de repenser la façon dont nous regardons le dessin d'un enfant pour qu'on puisse construire un environnement vraiment approprié pour son exploitation.

Nous relatons ici une expérience pratique avec le dessin développée lors de nos ateliers dans le cadre du projet Seconde période culturelle. Nous avons cherché à stimuler le regard, donc, la connaissance de ce qu'on voit. Nous avons utilisé le dessin d'observation en tant que processus constructif de répertoire. Nous avons constaté que les enfants avaient un univers de références graphiques très limité par des stéréotypes 


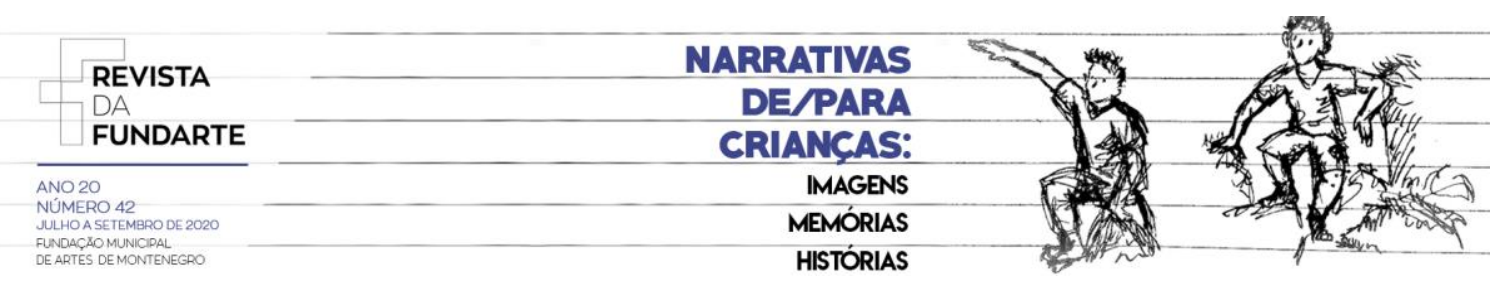

compris comme forme absolue dans la compréhension des images. La capture visuelle de la faune, de la flore, des bâtiments et des gens est souvent influencée par ce ce que nous connaissons déjà, c'est-à-dire, par ce qui a déjà été vu et emmagasiné dans le cerveau. Notre travail consistait à rendre le dessin un élément conducteur dans la création de récits, capable d'aider l'élève à développer aussi bien la vision spatiale que les capacités inventive, intuitive et imaginative.

Pour la présente analyse, nous avons choisi le travail développé dans l'École Julia Lopes et dans l'École Anne Frank. L'école Julia Lopes est située près du Morro dos Prazeres, un quartier à risque où une Unité de police pacificatrice (UPP) vient d'être installée. L'installation de ces UPP est un projet du Secrétariat de sécurité de l'État du Rio de Janeiro visant instituer des polices communautaires dans les bidonvilles comme un moyen de démanteler les gouvernements parallèles menés par les gangs du trafic de drogue. La guerre entre les gangs pour le contrôle des points de vente de drogues instaure une ambiance de peur et d'instabilité chez la population de ces zones. Les trafiquants sont des jeunes souvent cooptés par le trafic de drogue dans leur enfance. L'État quasiment absent dans les bidonvilles, les trafiquants jouent son rôle, pourvoyant le paiement de médecins, de médicaments, de subventions pour des pompes funèbres ou pour l'achat de matériel scolaire parmi d'autres services plus ou moins basiques. En retour, les trafiquants peuvent compter sur la complicité des habitants, qui retrouvent, dans cette connivence passive, un peu de paix. Ces habitants survivent entre les fusillades, puisque les conflits fréquents entre les gangs rivaux pour la domination des zones de trafic engendrent une guerre sans fin. Les enfants de ces bidonvilles sont soumis dès la naissance à cette réalité sanglante où des projectiles peuvent les trouver sur le chemin de l'école ou lorsqu'ils jouent dans les ruelles. Ces enfants sont ainsi dépossédés de leur enfance, des jeux et de la paix. Dans nos régions les plus pauvres, l'accord entre les miliciens, les trafiquants et la police est souvent l'unique issue.

L'arrivée des UPP n'apporte pas un véritable changement dans la vie des habitants. En effet, ces unités ne font que déterminer les accords entre les gangs et la Montenegro, p.01-20, ano 20, no 42, julho/setembro de 2020.

Disponível em: http://.seer.fundarte.rs.gov.br/index.php/RevistadaFundarte/index> 30 de setembro de 2020 


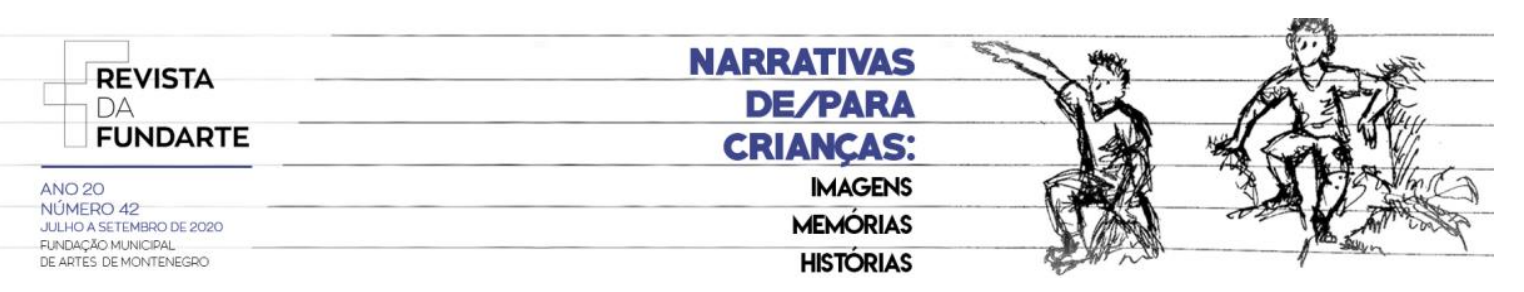

police. Le projet «Seconde période culturelle » proposait d'accueillir les enfants de ces bidonvilles à l'école en période intégrale`.

Le projet offrait de nombreux ateliers diversifiés. Les plus populaires étaient ceux de musique, de danse, de théâtre ou d'activités sportives, où le corps se détend du stress cumulé de manière plus immédiate. Des corps inquiets; beaucoup de mouvement; beaucoup de bruit et de la difficulté à se concentrer! Les corps voulaient libérer l'énergie contenue alors que le dessin exigeait de la respiration et de la retenue! Les séances duraient en moyenne trois heures, mais les premières 60 ou 90 minutes étaient consacrées à la concentration.

Norbert Elias (2000) indique, dans sa théorie du processus de civilisation, le développement conjoint de l'appareil psychique, dénommée psychogenèse, et les chaînes de relations sociales, la sociogenèse. Les modèles adoptés dans le processus de civilisation ne peuvent pas être perçus comme des entités abstraites, étrangères aux dynamiques concrètes propres à chaque période historique et à chaque société.

La civilité dans l'enfance est un processus de production symbolique. L'édition et la diffusion de livres ont contribué activement à la construction du concept de civilité, car elles visaient, à leurs débuts, à la diffusion de règles pour la vie en société. Cette production, qui se produit par l'intermédiaire de la lecture, meut un ensemble de dispositions intimes à l'imitation et à l'apprentissage qui serviront comme référence sociale. Dans le processus de lecture, les livres produisent des effets et des affects entre la jouissance du texte en tant que jeu et amusement et la lecture en tant qu'instruction. Les livres sont des objets précieux dans le mouvement de formation et d'intériorisation de l'expérience du monde. La création de livres avec les enfants serait ainsi un élément clé dans le processus de compréhension de la civilité au travers de la création d'un récit du contexte social où l'on s'insère. Ces récits dégagent les idées et

7 N.D.T. Au Brésil, on fréquente l'école pendant une seule période au choix : matin, après-midi ou soir. 


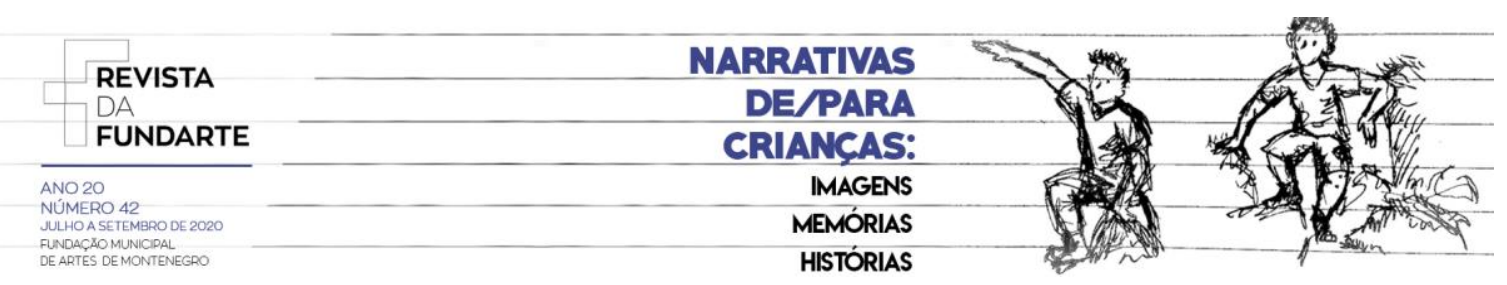

les valeurs imprégnées dans le conditionnement de l'autorisé et de l'interdit, qui influence intimement la structure des émotions et forme les sensibilités.

Contrairement à beaucoup d'ateliers, le nôtre posait le défi du silence et de la concentration. Nous consacrions une partie importante de la séance à rendre les enfants capables de se concentrer sur une activité durant plus de 10 minutes. Une petite distraction suffisait à faire échouer nos efforts: les élèves manquaient rapidement d'intérêt et pouvaient commencer à se battre, installant la pagaille. Au début de l'atelier, les matériels de dessin, limités, étaient souvent gâchés et les dessins mis au rebut comme s'ils n'avaient pour eux pas la moindre valeur. Puisqu'ils n'avaient pas accès à une meilleure compréhension du dessin, ils dessinaient de façon intuitive et rudimentaire - ce qui ne les intéressait pas. Lors d'une des séances, j'avais une théière que j'allais utiliser avec les étudiants de l'université l'après-midi et ai décidé de tester ce qui se passerait si je leur apprenais à observer et reproduire l'objet. J'ai donc rangé les pupitres autour d'une table, où j'ai posé la théière. Sur le tableau, j'ai fait un rectangle. J'ai commencé à conduire l'observation en dessinant la théière à l'intérieur de ce rectangle. Les enfants se sont intéressés et ont commencé à suivre progressivement les coordonnées. L'agitation s'est transformée en silence. Ils sont restés concentrés pendant 40 minutes. Ils se sont plongés en eux-mêmes!

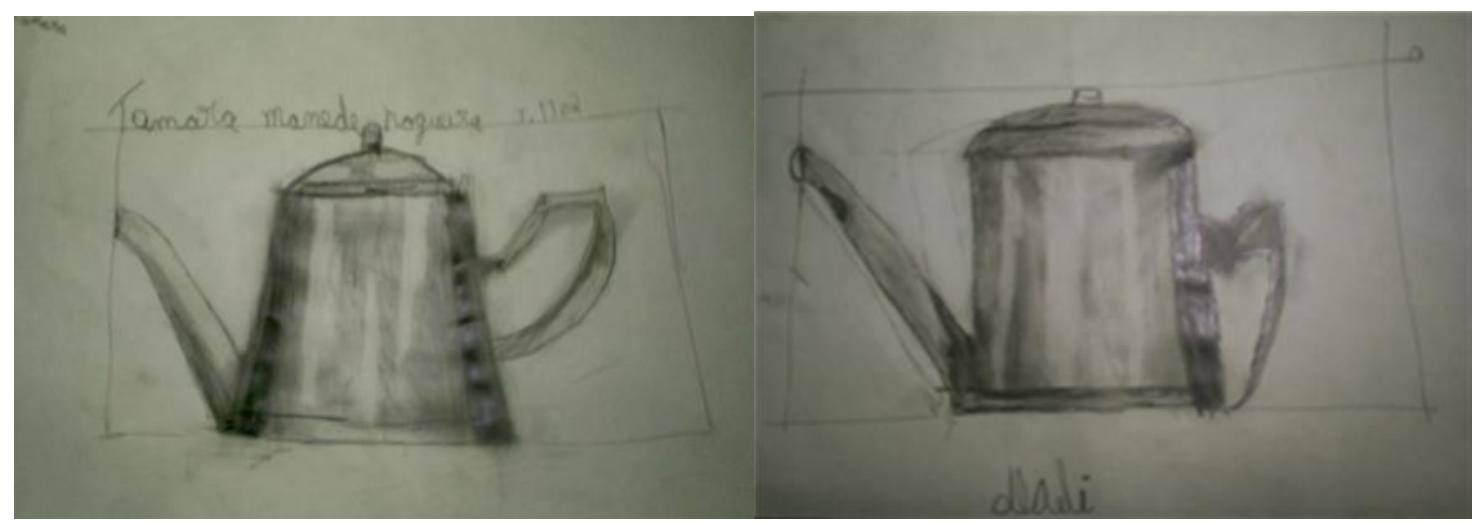

Images 1 et 2: Images produites par l'observation. Théières. (Source: Auteure) Montenegro, p.01-20, ano 20, no 42, julho/setembro de 2020.

Disponível em: http://.seer.fundarte.rs.gov.br/index.php/RevistadaFundarte/index> 30 de setembro de 2020 


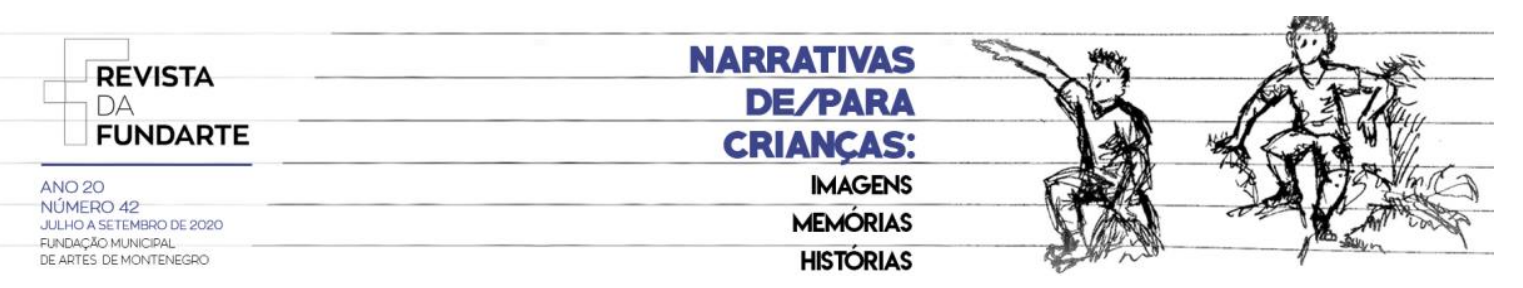

Le résultat a été surprenant aussi bien pour moi que pour les enfants. Ils ont été pris par une vague de bonheur face à la représentation faite, très proche de l'objet qu'ils voyaient devant eux. Fiers de leurs dessins, ils ont voulu les amener et les montrer à la directrice de l'école. Ce fut un véritable tournant dans leur intérêt et dévouement à l'atelier. La participation a accru et de nouveaux élèves sont apparus.

Dans le programme scolaire, le cours d'art est souvent relégué au second plan comme s'il était perçu comme inutile, sans valeur. De nombreux enseignants corroborent à l'entretien de cette stigmatisation puisqu'ils ne valorisent eux-mêmes pas la production des élèves. Le manque de préparation à la conduction d'un travail menant l'enfant à développer ses moyens d'expression favorise les idées reçues dans la construction d'un répertoire graphique. Apprendre à observer la forme et les différentes façons de la reproduire ne bride pas le processus créatif des enfants. II leur donne, au contraire, des outils. L'enfant est parfois aliéné de son propre potentiel, car soumis aux impositions des connaissances du professeur. Ce système confond la conduction, le soutien et la construction avec l'imposition. Structurant sa façon de voir et de reproduire le monde à l'aide de techniques et de matériaux diversifiés, l'enfant ne sera pas pris en otage par l'enseignement et par l'évaluation qui dénigrent et rabaissent leurs possibilités de subversion des couleurs, des formes, de création et de subjectivité. Ostrower observe que, à l'école, on ne tient malheureusement pas compte

de la créativité authentique, de la possibilité que chacun a de retrouver, dans les différents moments de son processus de création, sa propre mesure de ses capacités au sein de sa propre sensibilité, et la possibilité d'être évalué dans ce qu'on est vraiment et dans ce qu'on peut être. (OSTROWER, 1987, p 13-14.).

Dans la culture de l'image, la vue est à la sensation ce que le regard est pour à la pensée. Penser l'enfant en tant qu'être social, qui construit et qui crée de la culture, signifie défendre ses possibilités de raconter et de narrer le monde qui l'entoure. Montenegro, p.01-20, ano 20, ํo 42, julho/setembro de 2020. 


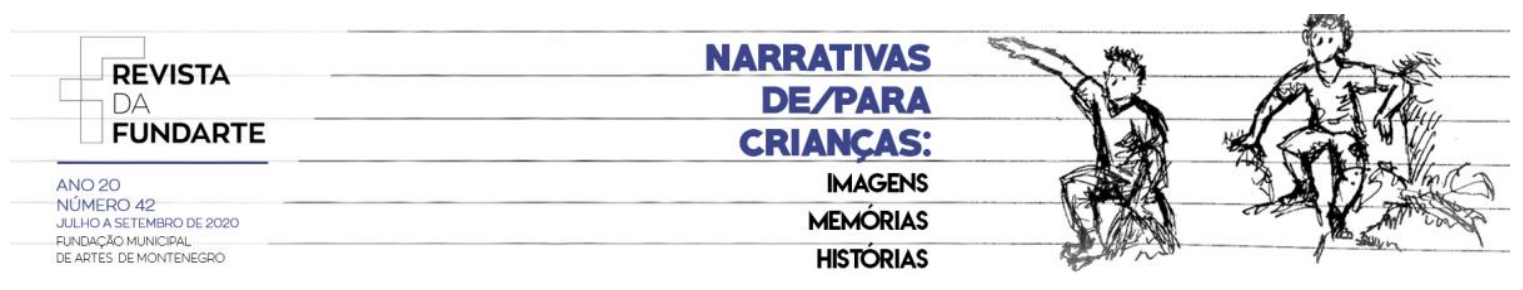

\section{Merveille mouvante - Observation et reproduction}

Dans la deuxième étape de l'atelier avec les élèves de l'École Julia Lopes, nous sommes allés à l'extérieur de l'école pour observer les arbres. Le but était d'échapper des lieux communs figurant dans les manuels scolaires, où les arbres sont quasi systématiquement des boules vertes à pois rouges représentant des pommes qui ne poussent même pas dans notre pays.

Rio de Janeiro possède une forêt dotée d'une faune et d'une flore riches et variées. Les observer afin de pouvoir les saisir est un acte d'amour que nous devons construire dès l'enfance. L'École Julia Lopes se trouve sur les pentes de la forêt tropicale, où des arbres séculaires étreignent la ville. Assis par terre avec leurs planches à dessin, les élèves ont entrepris l'observation d'un grand arbre en face de l'école.

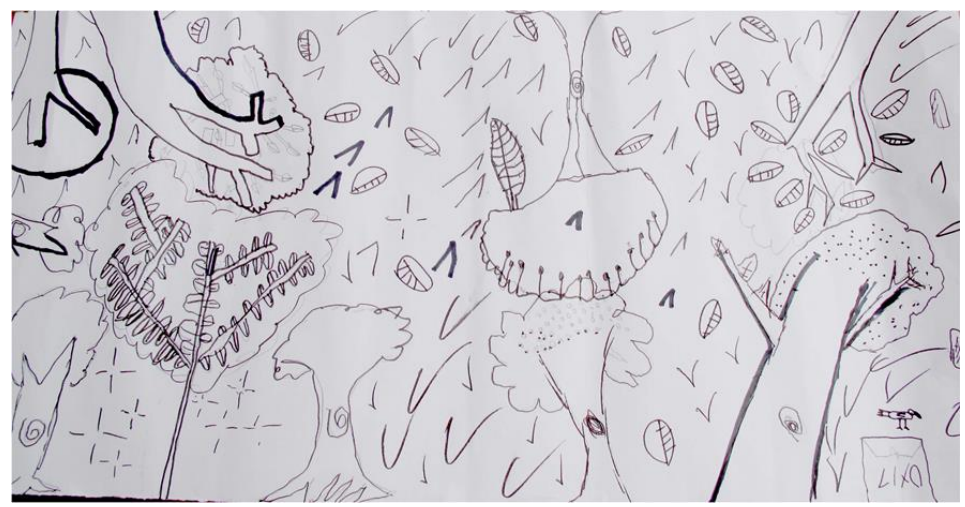

Image 2: Dessins d'observation des arbres. Crayon et stylos (Source: auteure)

Les dessins reproduisaient la structure de l'arbre avec le tronc, les branches, les feuilles et des textures - des dessins spontanés et expressifs. En classe, les enfants ont dessiné les feuilles séchées que nous avons ramassées. Chaque élève a ensuite découpé la sienne et ils ont organisé ensemble une composition en les collant sur une grande feuille. L'ensemble produisait une sorte de motif.

LIMA, Maria da Graça Muniz. C'est chaud...la création dans des zones dangereuses. Revista da FUNDARTE. Montenegro, p.01-20, ano 20, no 42, julho/setembro de 2020.

Disponível em: http://.seer.fundarte.rs.gov.br/index.php/RevistadaFundarte/index> 30 de setembro de 2020 


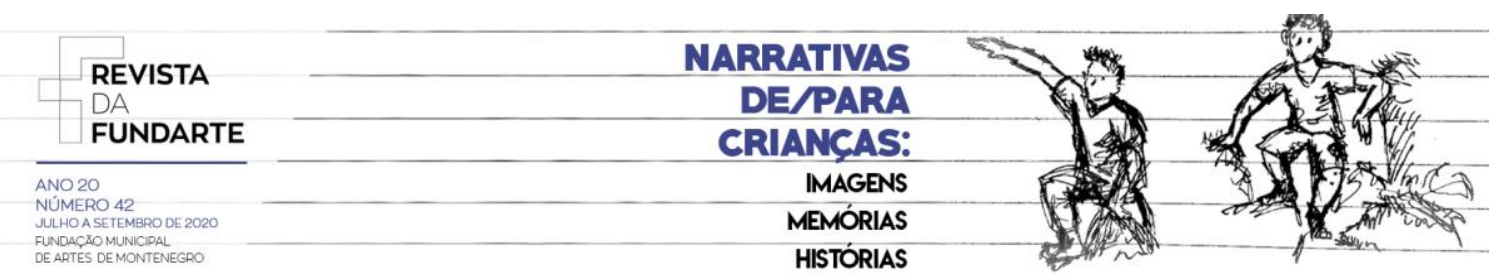

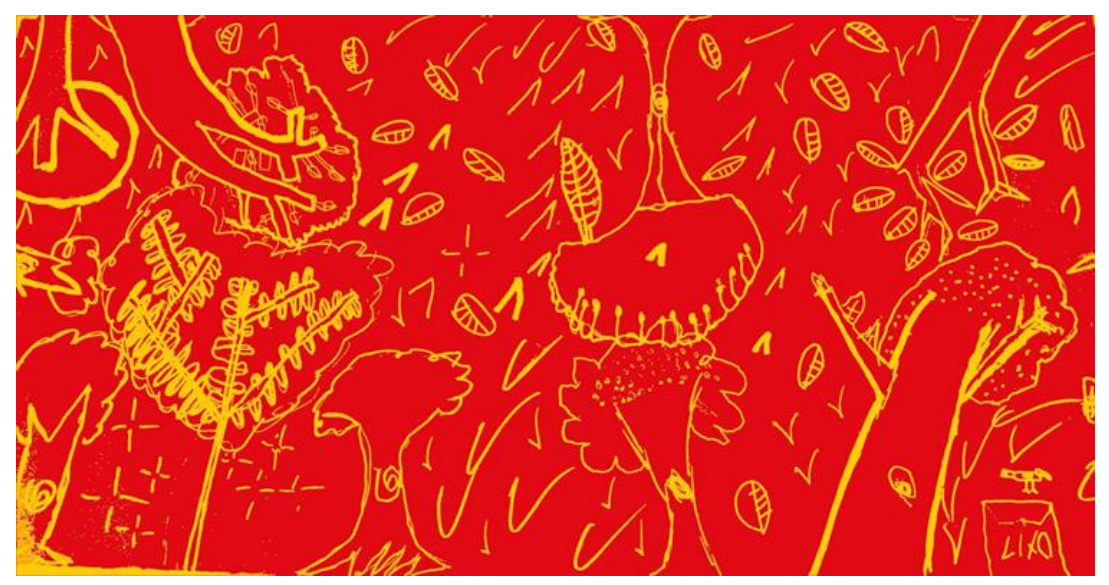

Image 3: Les dessins numérisés et coloriés sur ordinateur (Source: auteure)

Ils ont travaillé avec intérêt et curiosité. Ce motif de feuilles, d'arbres et d'autres dessins faits en classe a été numérisé et coloré sur ordinateur à une étape plus avancée de l'atelier.

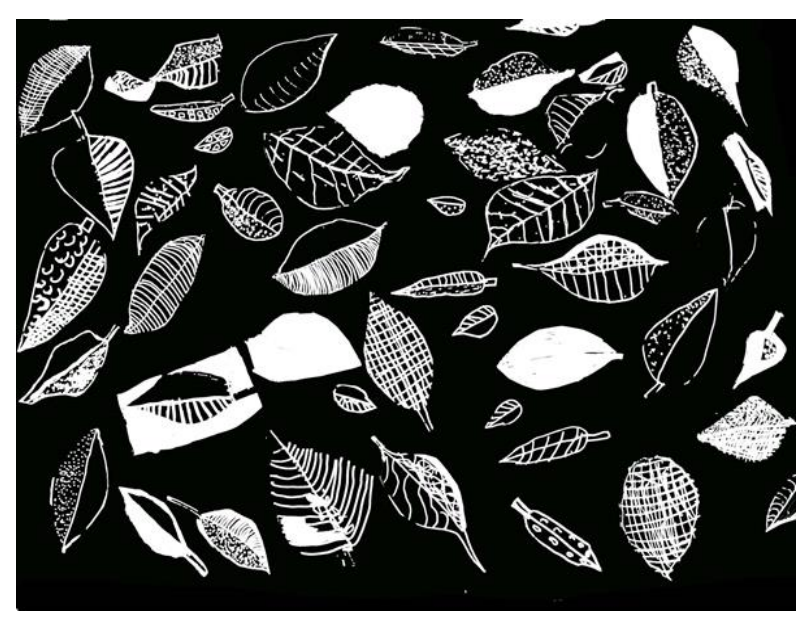

Image 4: Image 3: Les dessins numérisés et coloriés sur ordinateur (Source: auteure)

LIMA, Maria da Graça $N$ Montenegro, p.01-20, anc Disponível em: http://.see

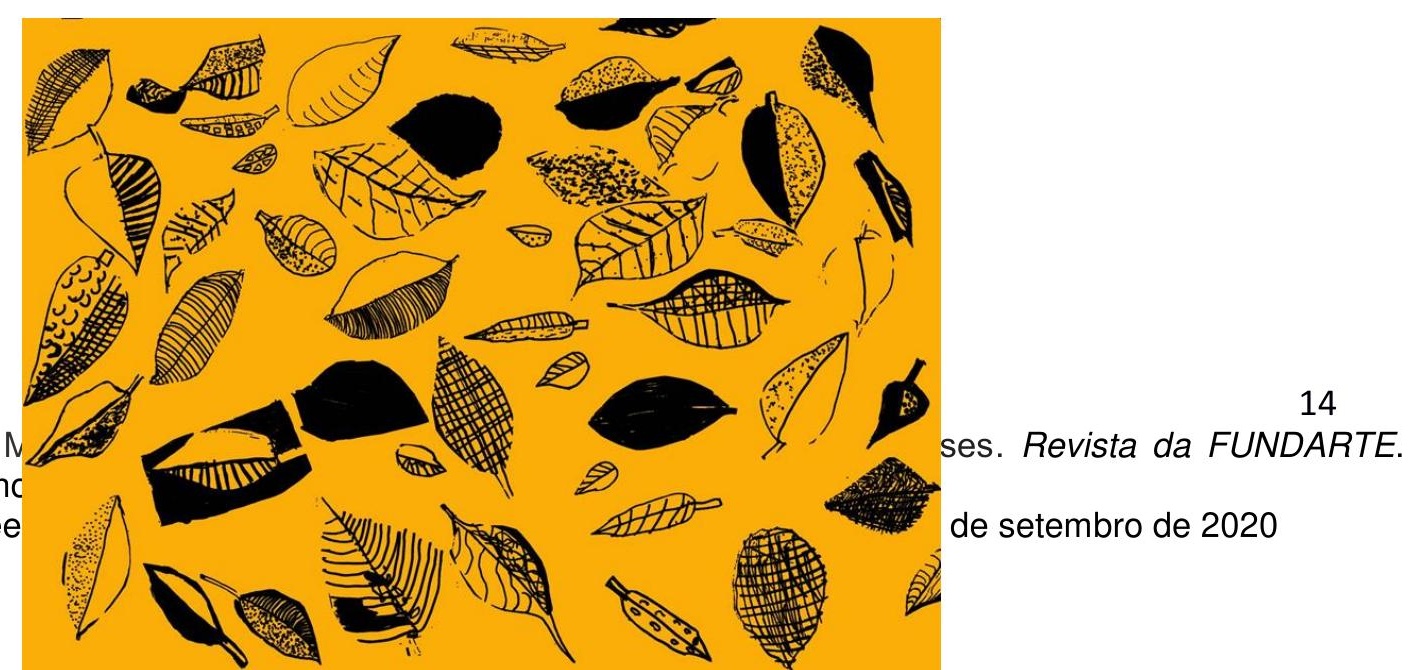




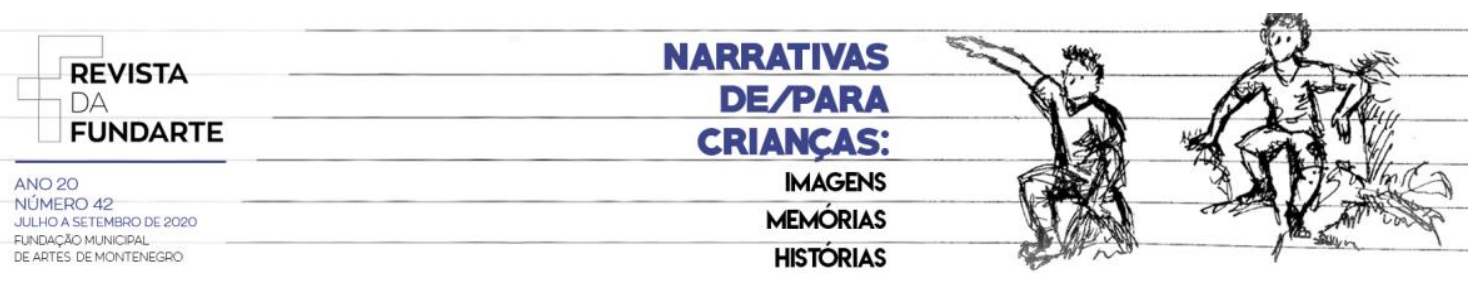

Image 5: Les dessins numérisés et coloriés sur ordinateur (Source: auteure)
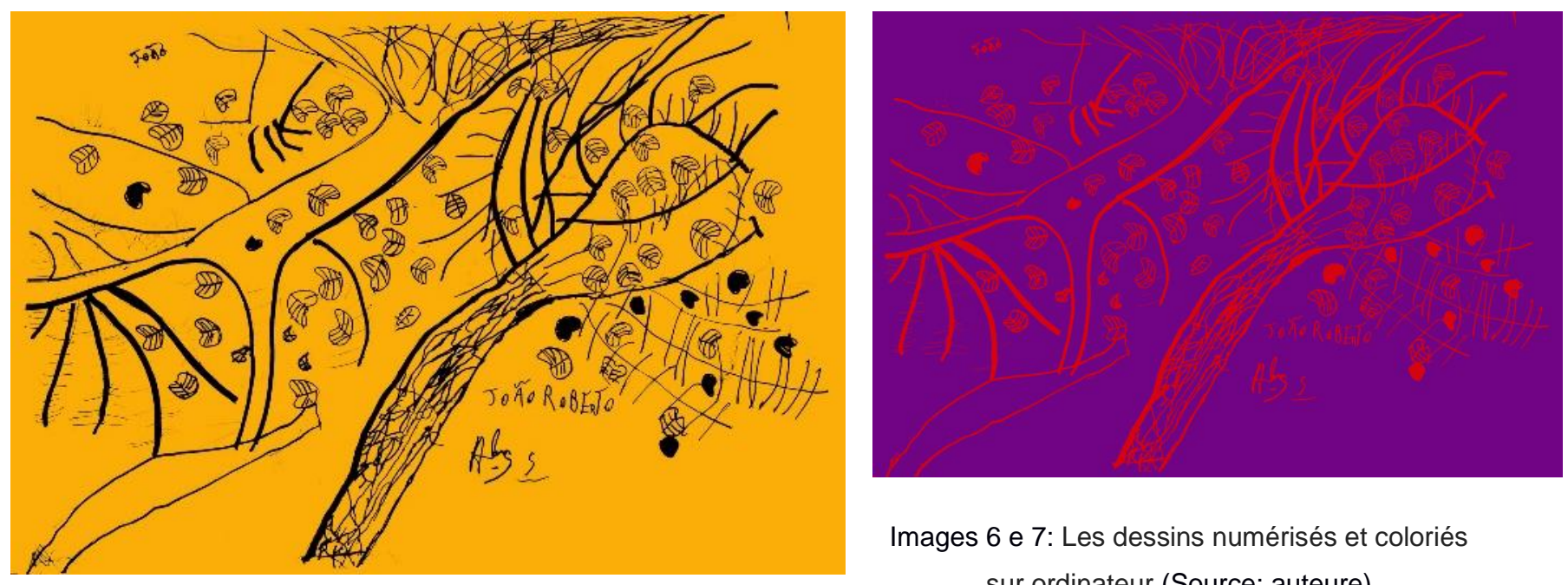

Images 6 e 7: Les dessins numérisés et coloriés sur ordinateur (Source: auteure)

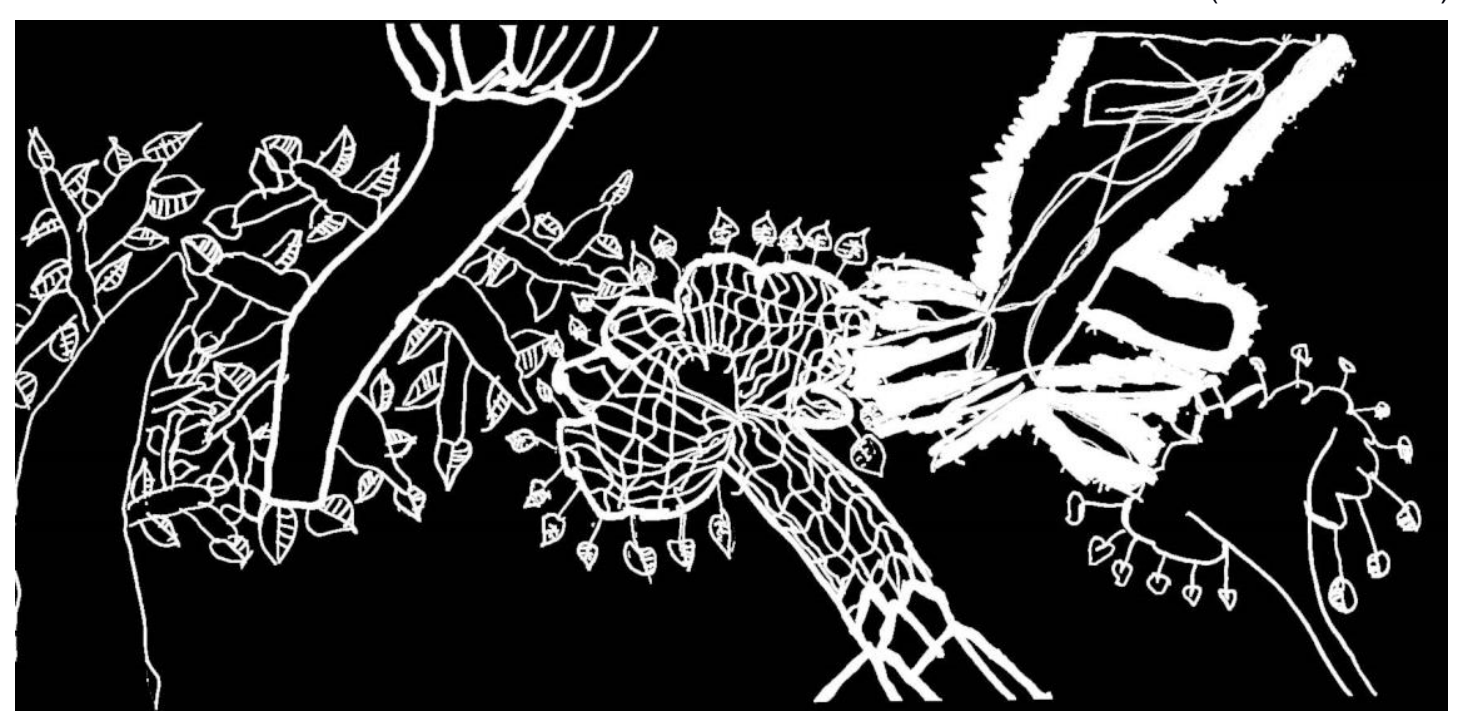

Image 8: Dessin numérisé et colorié sur ordinateur (Source: auteure)

LIMA, Maria da Graça Muniz. C'est chaud...la création dans des zones dangereuses. Revista da FUNDARTE. Montenegro, p.01-20, ano 20, no 42, julho/setembro de 2020.

Disponível em: http://.seer.fundarte.rs.gov.br/index.php/RevistadaFundarte/index> 30 de setembro de 2020 


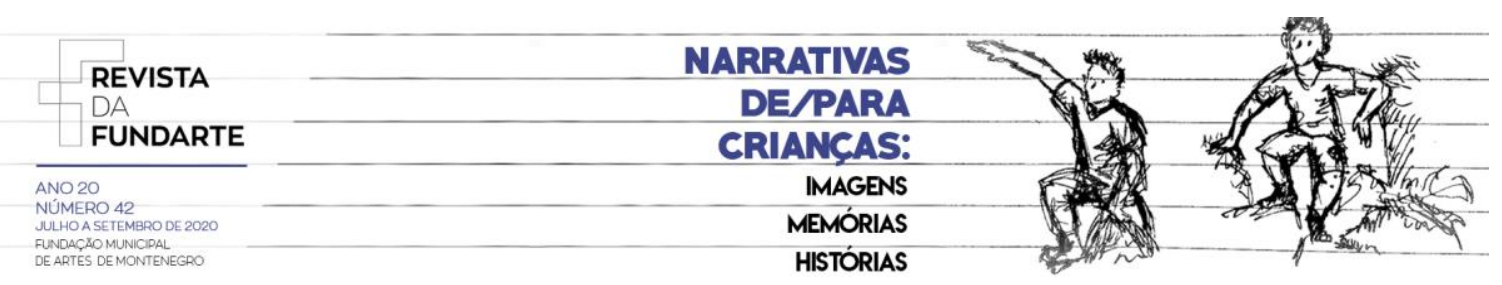

La découverte du potentiel de création du groupe a produit un émerveillement croissant - merveille mouvante - avec de nouvelles propositions. Le monde des formes et des couleurs qui les entourait intégrait désormais leur répertoire visuel en transformation.

\section{Rio est une ville de villes camouflées, avec des gouvernements mélangés, parallèles...}

Selon Abramovay (2005), il est possible d'affirmer que l'école n'est plus un espace protégé. Elle est devenue un lieu qui reproduit l'autoritarisme que nous retrouvons dans la société et encourage, à son tour, de multiples formes de violence. L'école peut certes être une victime de l'oppression, mais elle en est aussi l'auteure. En tant que victime, il est possible de considérer que la violence existe dans la société, indépendamment de l'école. L'auteure retrace une typologie des différentes formes de violence qui affectent l'environnement scolaire. La première, dénommée «la violence dans les écoles», est associée au trafic de drogue. Elle se manifeste dans la participation des élèves à des gangs, dans la vente et la consommation de drogues dans les bidonvilles et dans les conflits territoriaux des gangs.

À la dernière étape de l'atelier dans l'école Julia Lopes, nous avons entrepris la construction d'un récit qui se transformerait en un livre. Nous avons divisé les élèves en groupes de deux et avons fourni à chacun deux feuilles A4 reliées verticalement. Chaque élève observait son collègue afin de le dessiner, profitant de l'espace de la reliure des feuilles. Dans un premier temps, ils éprouvaient beaucoup de difficultés à observer les traits de l'autre. Ils riaient, bougeaient et provoquaient le collègue. Être avec soi-même et avec l'autre tout en faisant attention à la spatialité n'était pas facile. Si l'observation était difficile, reproduire ce que l'on observait sur les deux feuilles reliées l'était aussi. Quelques dessins débordaient les limites des deux feuilles alors que d'autres n'en occupaient même pas l'espace d'une. Les travaux ont été achevés avec l'utilisation de collages, crayons de couleur et feutres, et ont ensuite été numérisés. 


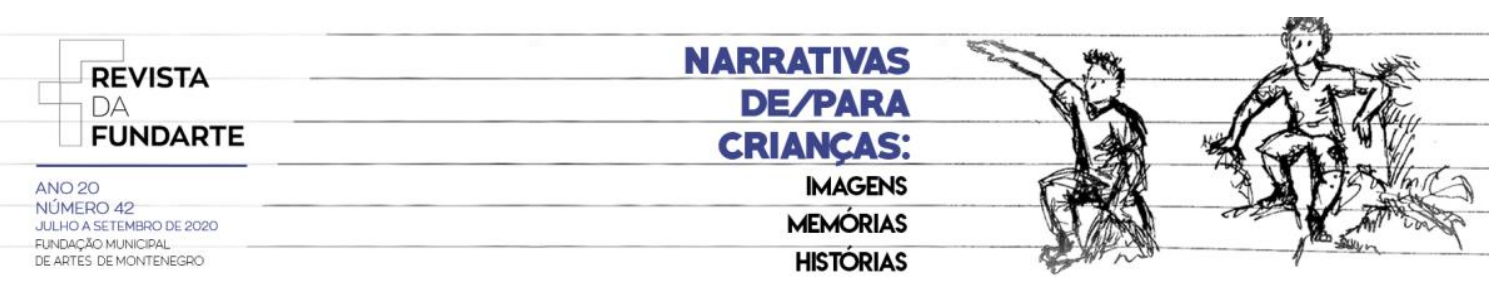

Nous avions un groupe de personnages pour la construction d'un récit. Les dessins ont été réduits au format du livre, coupés en bandes, pliés en deux et collés sur la page. La face pliée était destinée au dessin de la maison de chaque personnage. Les personnages et ses décors respectifs étant prêts, nous étions prêts à créer notre récit.

Bruner (1987) estime que les façons de raconter le vécu et les moyens de conceptualiser des enfants deviennent tellement habituels qu'ils constituent des formes de structuration de l'expérience. Selon lui, ces formes narratives organisent et fournissent le contexte approprié pour rendre compte des témoignages des expériences personnelles. Elles déterminent quels aspects de l'expérience vécue seront exprimés et quels sens y seront attribués par ses créateurs.

Avec tous les personnages créés et leurs foyers respectifs, nous avions les protagonistes et un public. J'ai demandé aux étudiants d'expliquer qui étaient ces personnages, ce qu'ils faisaient dans la vie, où ils habitaient et quelles seraient leurs histoires. L'un des étudiants, un garçon très proactif, a rattaché trois ou quatre feuilles et a déterminé que l'histoire serait celle d'un dragon. II s'est mis à dessiner et a rapidement été suivi par le groupe, excité par l'image fantastique d'un dragon. Pendant qu'ils dessinaient, ils ont commencé à raconter l'histoire :

" Il était une fois une ville où vivaient beaucoup d'honnêtes gens qui travaillaient dur. Ils étaient tristes et effrayés par un dragon, qui troublait leur paix. Chaque jour, le dragon apparaissait dans les rues et crachait des boules de feu, brûlant les maisons et les gens. Les gens, vraiment effrayés, décidèrent de se réunir pour parler au dragon, qui vivait dans une montagne voisine. En arrivant à la maison du dragon, tout le monde avait peur de ce qui se passerait. Ils appelèrent :

- Dragon! Dragon! Dragon!

- QUI EST-CE ? ! ! ! ! ! ! ! ! ! ! ! - hurla le dragon en sortant et crachant du feu.

Effrayé, le groupe dit :

- Nous sommes venus savoir pourquoi vous n'arrêtez pas de nous faire peur et de cracher du feu dans la ville. 


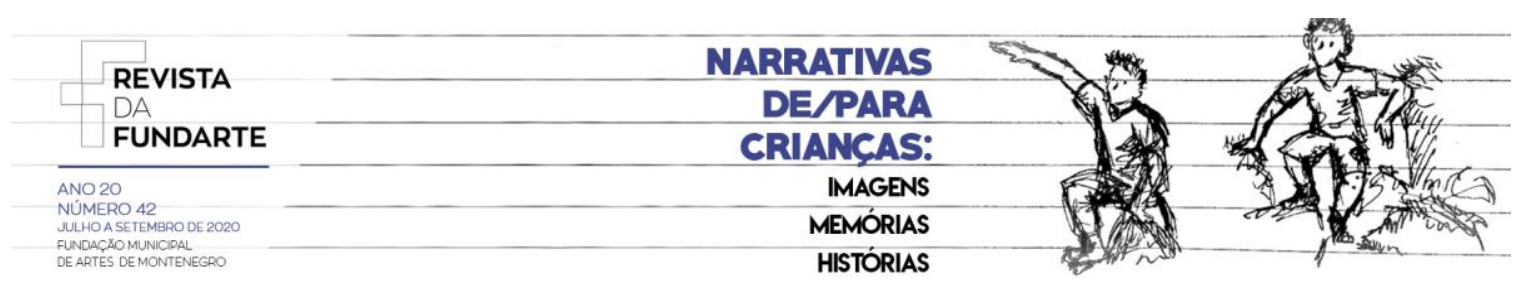

- J'ai besoin de manger, répondit le dragon. J'ai un chiot et vais en ville pour m'approvisionner.

Le groupe répondit :

- Nous voulons vous proposer un marché.

- Quel marché ? - demanda le dragon.

- Si nous vous apportons de la nourriture tous les jours, vous nous laisseriez tranquilles ? - répondit le groupe. »

Content, le dragon accepta l'accord.

Ils se mirent d'accord sur la façon dont la nourriture serait livrée et ils vécurent heureux à jamais.

Les dessins ont été achevés; les pages du livre assemblées avec la couverture et l'atelier finalisé. Le dragon qui occupait la communauté a reçu sa partie de l'accord de commandes parallèles et les habitants suivaient avec des intervalles de paix dans la guerre camouflée.

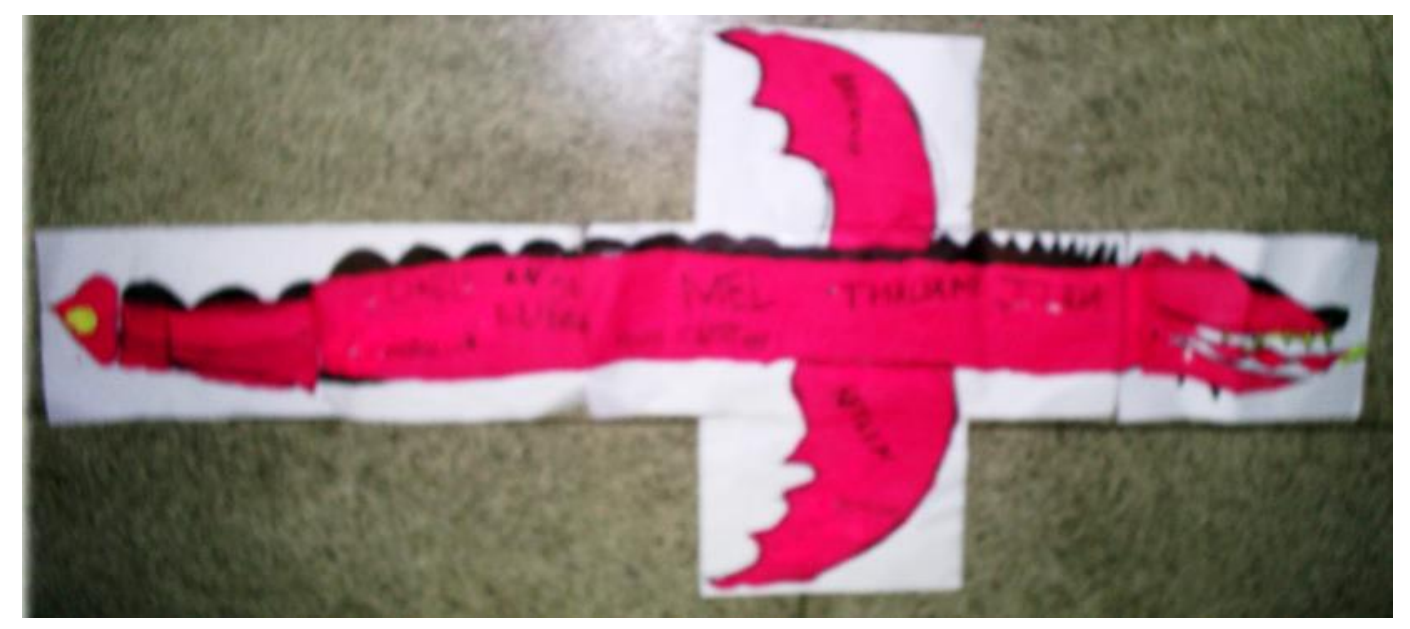

Image 9: Dessin de personnages. Techniques mixtes

Nous pouvons établir un parallèle avec l'atelier que nous avons développé à l'École Anne Frank, qui - nous le réitérons - est située près du Palais Guanabara, dans le quartier Laranjeiras, dans la zone sud de la ville de Rio de Janeiro. Les élèves 


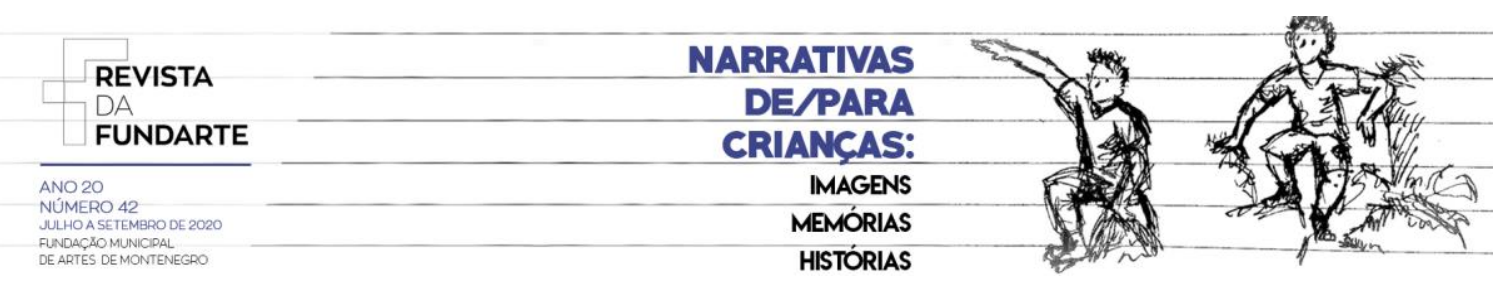

sont issus de familles de travailleurs de base de la région : des concierges, femmes de ménage, vendeurs, entre autres. Ils appartiennent à une classe sociale de salariés à revenus intermédiaires et faibles vivant dans les limites du quartier, incrusté dans une zone aisée de Rio. Dans cette école, les procédures pour l'élaboration de l'atelier étaient les mêmes ; toutefois, le récit résultant a été très différent.

L'histoire racontée par les enfants tournait autour de préparatifs pour l'ouverture d'une boîte de nuit à Barra da Tijuca, quartier nouveau riche de la ville. La structure des foyers des personnages présentait des caractéristiques comme des baignoires en or, des piscines, plus d'une voiture et vue sur la mer. Les personnages engageaient des conversations frivoles sur des vêtements, du maquillage, des coiffures et des commérages de presse people... Leur récit ressemblait beaucoup aux telenovelas brésiliennes diffusées sur les chaînes de télévision ouvertes. Ces émissions cultivent le glamour de la vie des couches les plus riches de la société.

L'espace de l'imaginaire narratif est clairement imprégné du contexte socioculturel.

Nous constatons que, malgré la difficulté du quotidien des enfants des quartiers à risque, il y existe un champ complexe de forces. La découverte et la réalisation de contenus artistiques amènent à une émancipation de récits par rapport au circuit imposé par les médias de masse.

L'expérience esthétique nous donne accès à l'autre, comme un moyen d'ouverture à l'altérité, nous y rendant ainsi plus sensibles. L'esthétique stimule notre capacité à saisir la réalité par les voies de la sensibilité.

\section{Referências :}

ABRAMOVAY, M. (Org.). Cotidiano das escolas: entre Violências. Brasília: UNESCO; Observatório de Violências nas escolas; MEC, 2005.

BRUNER, J. Atos de significação. Porto Alegre: Artes Médicas, 1997.

CASSIRER,Ernst. Ensaio sobre o homem. São Paulo: Editora Wmf Martins Fontes, 2005. 


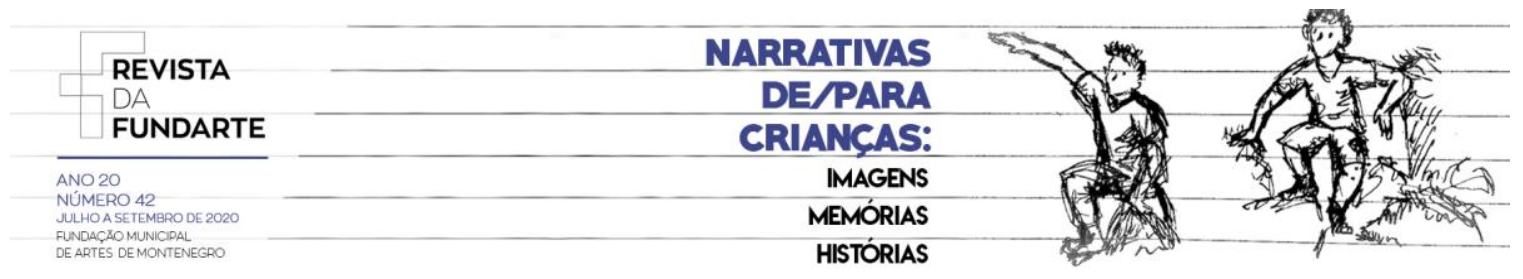

ELIAS, Nobert. The civilizing process: sociogenetic and psychogenetic investigations. Massachusetts: Blackwell, 2000.

OSTROWER, Fayga. Criatividade e processos de criação. Petrópolis: Vozes, 1987.

VIGOTSKI, L. S. Imaginação e criação na infância. São Paulo: Ática, 2009. Montenegro, p.01-20, ano 20, no 42, julho/setembro de 2020. 